
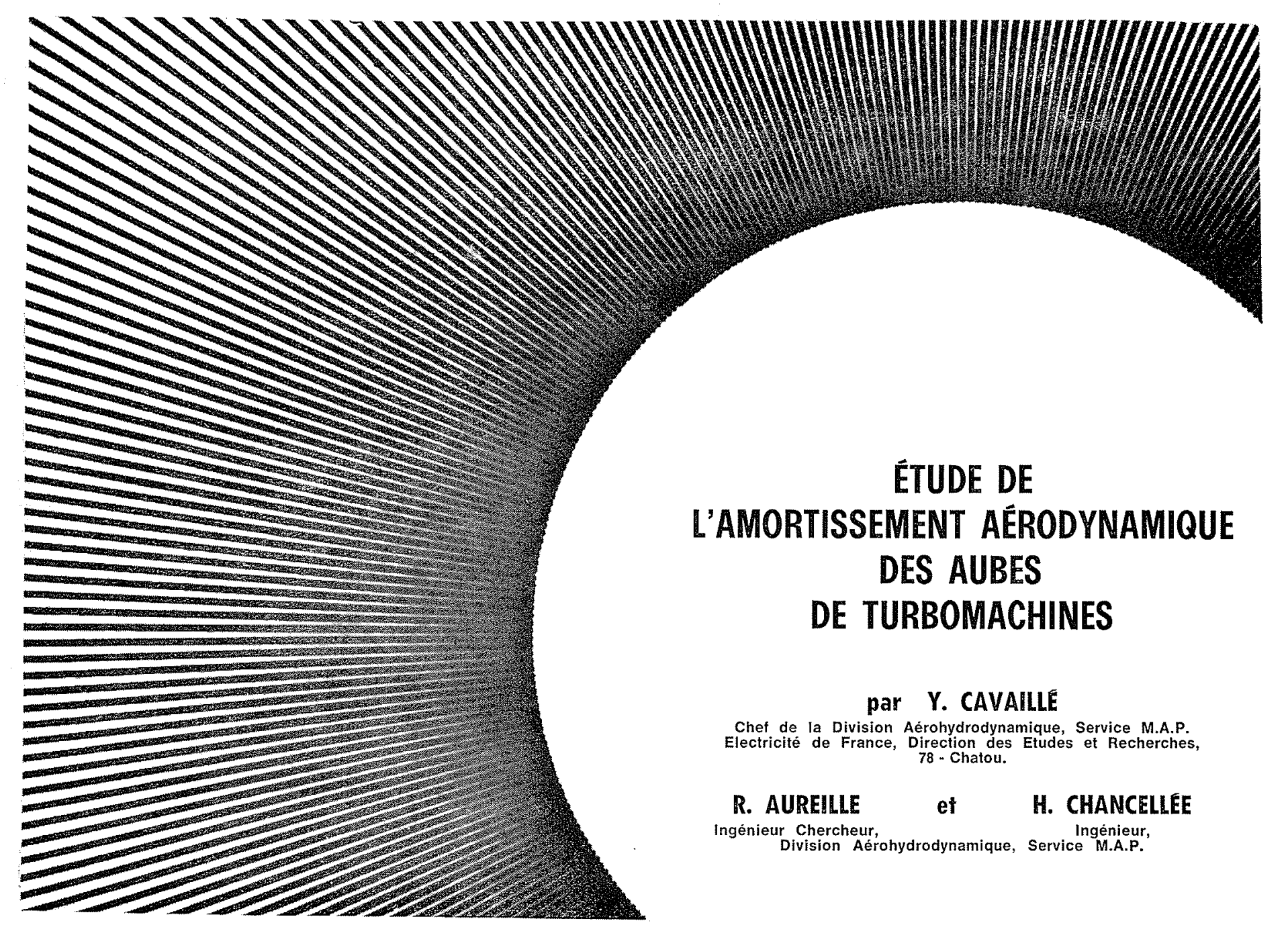

Introduction

Les aubes de turbomachines sont soumises à des excitations d'origines diverses qui les font vibrer. Ces excitations peuvent être d'ordre mécanique (défaut d'équilibrage, vibrations des disques, vibrations des viroles...) ou d'ordre hydro (ou aéro) dynamique (décollements, ondes de pression et surtout pertubations apportées par les sillages d'un aubage sur l'aubage suivant). En particulier, si l'on considère de façon schématique le cas des pales d'une roue mobile, celles-ci sont soumises à des forces constantes pendant leur passage dans les portions d'écoulements uniformes issues des canaux inter-aubes du distributeur mais, pendant la traversée des sillages, les forces varient considérablement en direction et en module. Chaque pale est ainsi soumise à une force excitatrice périodique due à l'intersection des sillages de l'aubage amont. Bien sûr, il est possible de limiter l'importance de ces excitations en soignant la réalisation mécanique, en particulier l'équilibrage et en augmentant la distance qui sépare les aubages de telle sorte que les sillages soient atténués. On peut aussi éviter les risques de résonance en réalisant des pales et des structures dont les fréquences propres soient différentes de celles des principales forces excitatrices, mais, en définitive, il y aura toujours des forces excitatrices plus ou moins intenses qui feront vibrer les aubes à la résonance ou en régime de vibrations forcées et même si les déformations et les contraintes semblent acceptables, il convient néanmoins de savoir si le mouvement vibratoire des aubes, aussi petit soit-il, est susceptible d'être amorti ou, au contraire, amplifié par l'écoulement du fluide à travers la tur-

\section{ÉTUDE DE \\ L'AMORTISSEMENT AÉRODYNAMIQUE DES AUBES DE TURBOMACHINES}

par Y. CAVAILLE

Chef de la Division Aérohydrodynamique, Service M.A.P. Electricité de France, Direction des Etudes et Recherches, 78 - Chatou.

\author{
R. AUREILLE \\ et \\ H. CHANCELLEE \\ Ingénieur Chercheur, \\ Ingénieur, \\ Division Aérohydrodynamique, Service M.A.P.
}

bomachine. Il importe non seulement de savoir si des conditions stabilisatrices sont obtenues au point de fonctionnement nominal, mais il faut en outre s'assurer que ce résultat reste acquis pour tous les régimes de fonctionnement et en particulier pour les conditions de fonctionnement hors adaptation.

Définition

de l'amortissement aérodynamique d'un profil d'aube isolé

Pour définir ce que nous appellerons conventionnellement le phénomène " d'amortissement aérodynamique », considérons un profil d'aube isolé placé dans un écoulement uniforme horizontal. La force aérodynamique de portance qui s'exerce sur une tranche de profil de longueur unité est verticale et a pour valeur $\mathrm{P}_{0}=1 / 2 \mathrm{\rho V}_{0}{ }^{2} \mathrm{C}_{z} \times l$.

Si on fait varier l'incidence du profil on obtient des portances (et des coefficients $\mathrm{C}_{z}$ ) différentes qui permettent de tracer la polaire $\mathrm{C}_{z}=\mathrm{C}_{*}$ (i) qui est une donnée caractéristique du profil considéré.

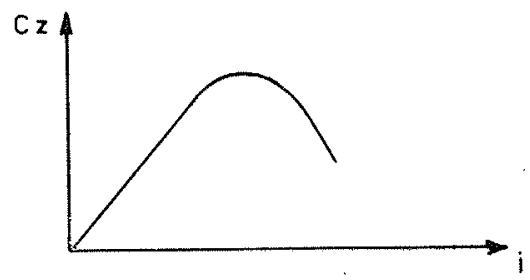




\section{Y. CAVAILlé, R. AUReille et H. ChanCellee}

Si nous considérons à présent le profil animé d'un mouvement vibratoire de translation vertical et si pour la simplicité du raisonnement nous faisons l'hypothèse que chaque position du profil est en état d'équilibre (hypothèse de quasi stationnarité), on voit alors qu'à chaque instant $t$, le profil a une vitesse instantanée $\mathrm{U}(t)$ et il est soumis à la portance $\mathrm{P}(t)$ perpendiculaire à la vitesse $\mathrm{W}(t)$ de l'écoulement relatif par rapport au profil.

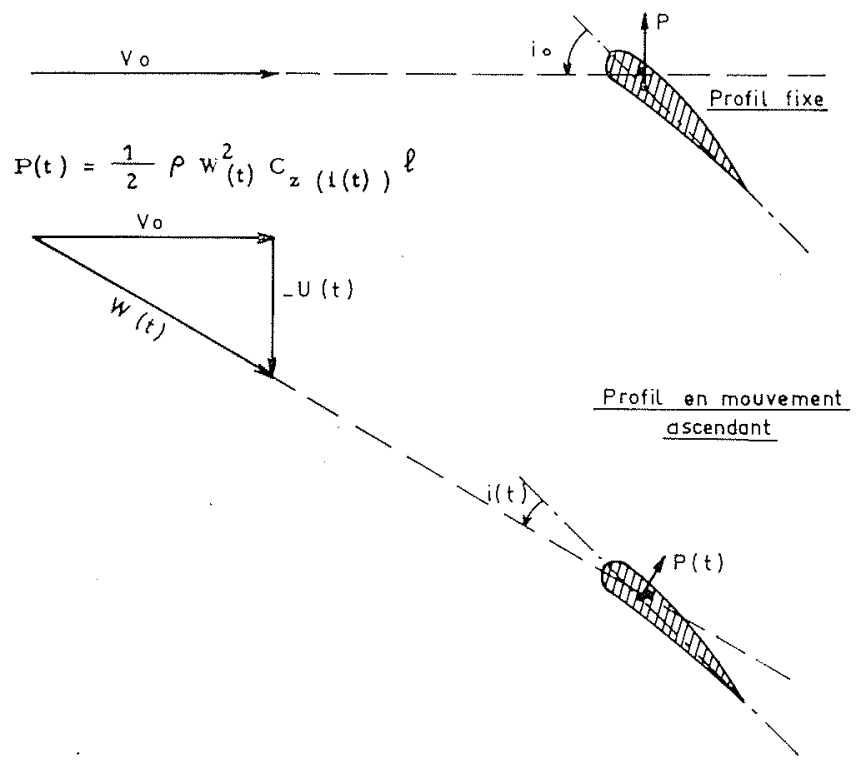

II apparaît donc que dans le domaine où la portance est une fonction croissante de l'incidence, lorsque dans son mouvement vertical le profil monte, sa vitesse instantanée se retranche de la vitesse de l'écoulement uniforme $V_{0}$ pour donner une vitesse relative $\mathrm{W}(t)$ dont la direction fait avec la corde du profil un angle $i(t)$ qui diminue la portance et s'oppose au mouvement. De même, lorsque le profil descend, la vitesse W $(t)$ donne un angle $i(t)$ qui augmente la portance et s'oppose al mouvement. Ainsi, lorsque le profil vibre, la portance quasistationnaire tend à le ramener au repos. Ce phénomène reste encore vrai [1] lorsque l'on considère les forces réellement instationnaires qui agissent sur le profil d'aile et c'est ce que nous appelons: « amortissement aérodynamique ».

Remarquons toutefois que, dans le domaine où la portance diminue lorsque l'incidence augmente, le résultat est inversé : il y a auto-excitation du profil placé au sein de l'écoulement. Dans ce cas l'hypothèse de quasistationnarité n'est plus valable en fluide réel [2] et il s'agit du phénomène dit «flottement de décrochage » ou « Stall flutter ». Ce phénomène n'est à redouter que lorsqu'une turbomachine fonctionne avec des aubes pouvant être en régime de décrochage.

\section{L'amortissement aérodynamique dans les turbomachines}

D'après les résultats relatifs aux profils d'aubes isolés, on peut être tenté de supposer que les phénomènes se passent de façon identique pour les profils d'un aubage de turbomachine. Cette approximation est sans doute valable lorsque le pas relatif des aubes est très grand mais il semble a priori douteux d'assimiler un profil d'aube isolé à un élément de grille d'aubes très serrées, donnant une forte déviation à l'écoulement et où le déplacement de chaque aube modifie les conditions d'écoulement autour des aubes voisines. Malheureusement la théorie est très compliquée [3] dans le cas d'une grille d'aubes et il est très difficile de prévoir exactement l'évolution des forces instationnaires qui s'appliquent sur les aubes d'une grille vibrant d'une façon quelconque au sein d'un écoulement. Cependant, certaines théories simplifiées [4] et les études expérimentales de Bellenot et Lalive d'Epinay [5] montrent qu'il n'est pas exclu que dans certains cas l'amortissement aérodynamique puisse décroître et même devenir négatif pour les aubes montées en grille.

Nous avons vu dans l'introduction que les aubes de turbomachines vibrent sous l'action des perturbations de l'écoulement; les amplitudes vibratoires dépendent fortement de l'amortissement mécanique et surtout aérodynamique, de sorte que même si au point de fonctionnement nominal ces vibrations d'aubes sont tout à fait acceptables, il importe quand même de s'assurer, en vue d'une meilleure fiabilité, que pendant les fonctionnements transitoires (arrêt, démarrage...) ou au cours de régimes peu fréquents correspondant à une marche hors adaptation (virage, minimum technique, admission d'air au condenseur pour les turbines à vapeur, marche à très fort débit sur chaudière éteinte pour les ventilateurs de souffage...), l'amortissement aérodynamique ne devienne pas négatif ou diminue trop rapidement et atteigne une valeur trop faible pour limiter à un niveau acceptable les contraintes vibratoires dues aux forces excitatrices.

Etudes faites ò̀ l'A.T.T.A.G.

Dans une première étape, une étude expérimentale a été entreprise en 1964 avec la collaboration du Laboratoire de l'A.T.T.A.G. (Association Technique pour la Turbine à Gaz). Cette étude avait pour but de mettre en évidence l'évolution de l'amortissement aérodynamique en fonction des conditions vibratoires et de l'écoulement.

Il est tout de suite apparu [6] que l'amortissement aérodynamique (planche 1) était très largement supérieur à l'amortissement mécanique ( 15 fois plus grand pour une période réduite VT/L égale à 127 , dans le cas d'une aube droite interactionnée par la veine d'essai). Cependant l'évolution de l'amortissement aérodynamique en fonction de la période réduite présentait un maximum qui pouvait laisser présager une diminution importante de son module, pour des valeurs suffisamment grandes de la période réduite. D'autre part il apparaissait (planche 2) que la disposition géométrique des aubes (inclinaison et pas relatif) était un paramètre important dans l'étude de l'amortissement aérodynamique.

On a ensuite entrepris d'étudier l'influence du rapprochement des fréquences propres de fiexion et de torsion sur le mouvement vibratoire et l'amortissement d'une aube placée dans un écoulement. Les résultats obtenus [7] montrent que lorsque les valeurs relatives des fréquences propres sont assez différentes $(11 \mathrm{~Hz}$ et $16 \mathrm{~Hz})$ l'amortissement aérodynamique est positif (planche 3) et crô̂t en fonction de la période réduite, dans le domaine exploré. Lorsque les valeurs relatives des fréquences propres de flexion et de torsion sont voisines $(90 \mathrm{~Hz}$ et $100 \mathrm{~Hz})$, on observe que l'amortissement évolue de façon irrégulière en fonction de la période réduite et, si l'on modifie convenablement la répartition massique de la pale (en ajoutant une faible masse en bout de pale par exemple), on peut obtenir [8] le phénomène de flottement (planche 4). Si- 
gnalons que l'apparition du flottement est très liée à la répartition massique, à l'écart relatif initial entre les fréquences propres de flexion et de torsion, au pas relatif des aubes, à la période réduite, etc.

\section{Etudes effectuées sur une pale de ventilateur en rotation}

Afin de confirmer d'une façon globale et dans les conditions réellement rencontrées sur une turbomachine, les résultats qui avaient été obtenus à l'A.T.T.A.G., on a entrepris d'étudier l'évolution de l'amortissement aérodyna- mique d'une pale de ventilateur en fonction de différents paramètres :

- vitesse de rotation de la machine;

- calage des pales de la roue;

- point de fonctionnement du ventilateur;

- fréquence propre de la pale.

Les mesures relatives à l'amortissement sont effectuées essentiellement sur une pale équipée de jauges de contraintes et excitée par une impulsion qui la fait vibrer en flexion, l'impulsion étant obtenue au moyen d'une charge de poudre dont la mise à feu peut être commandée. Les signaux issus des jauges ainsi que leurs alimentations et la commande de l'impulseur à poudre traversent un contact tour-
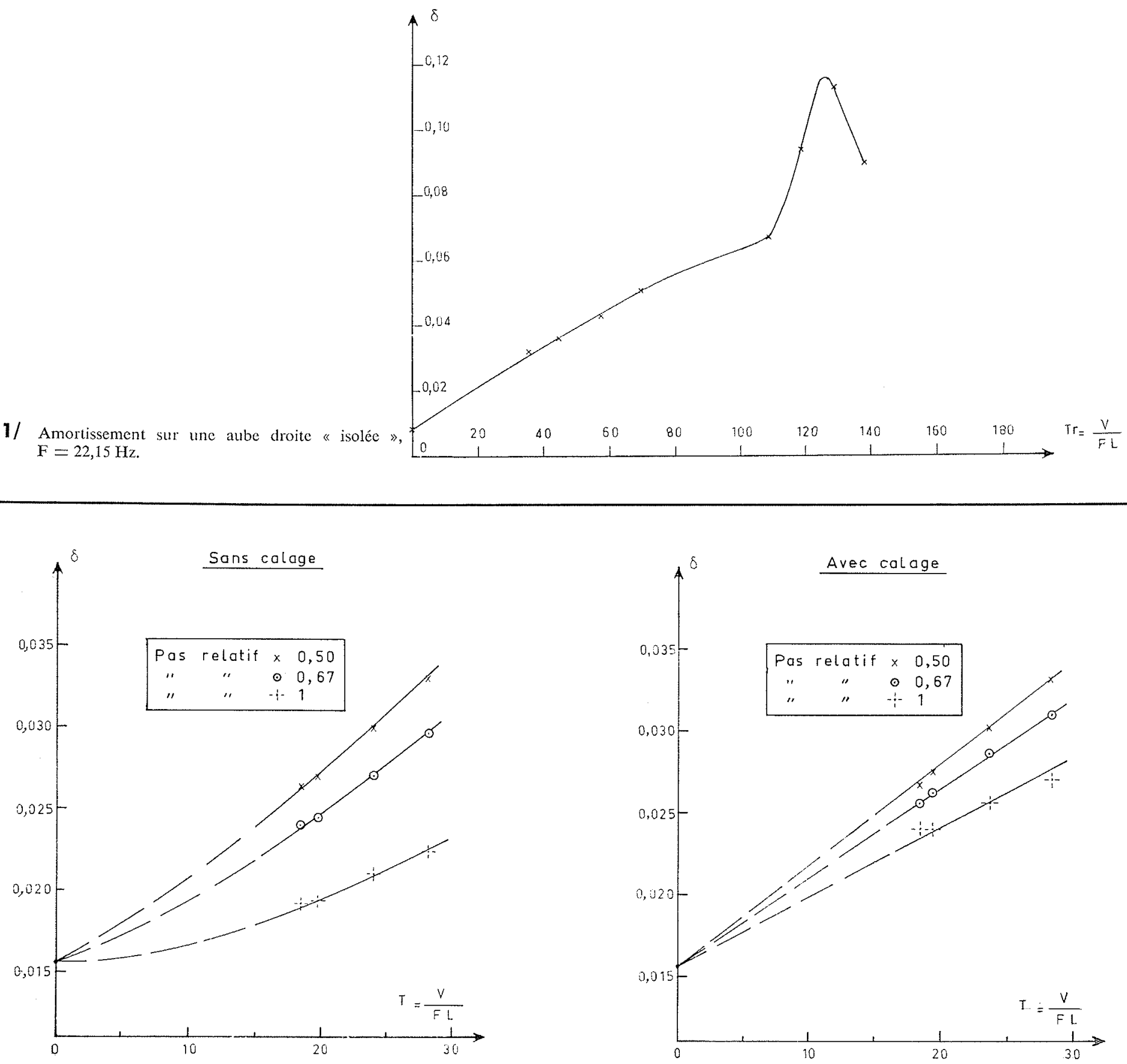

2/ Influence du calage et pas relatif sur une aube plane montée en grille inactive, $F \simeq 102 \mathrm{~Hz}$. 


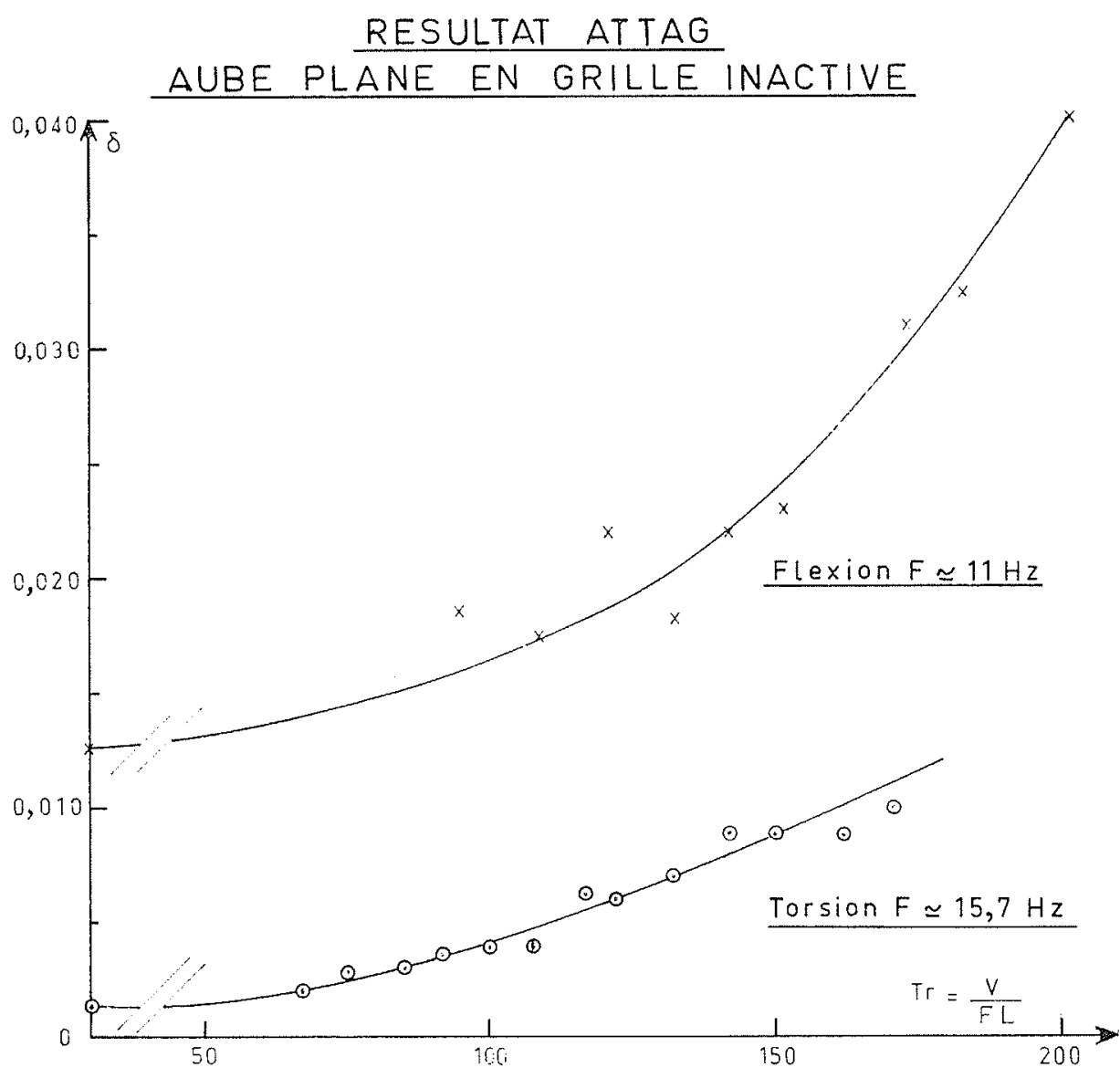

\section{AUBE CAMBREE EN GRILLE ACtIVE}

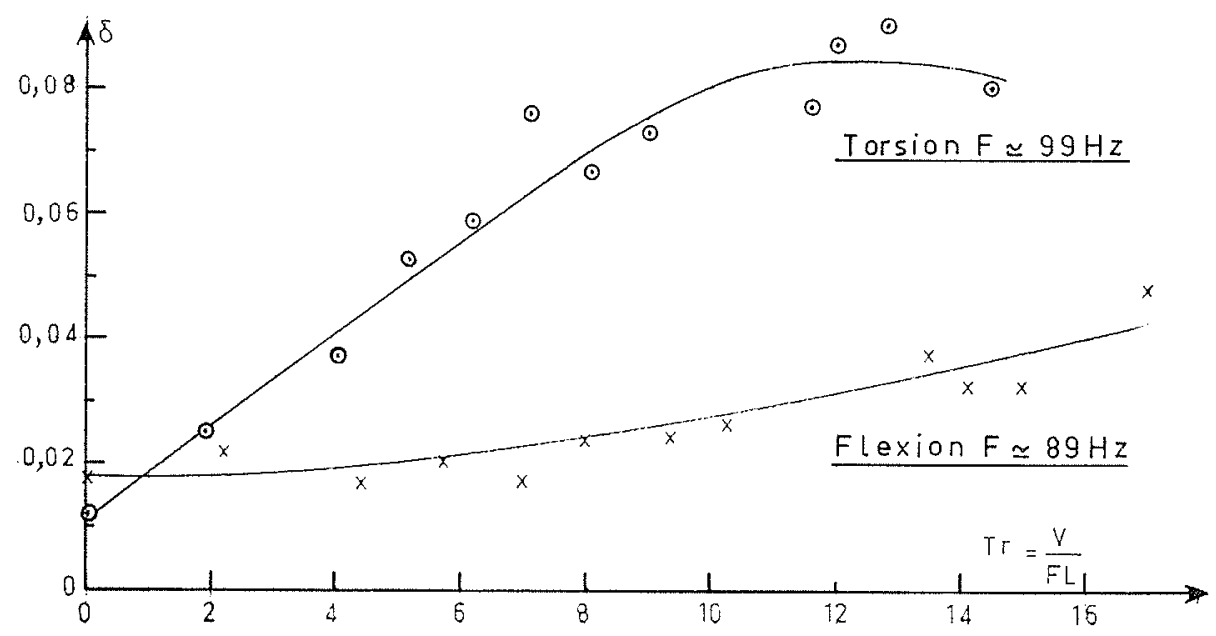

$3 /$ nant à bagues et balais qui permet d'effectuer les réglages et l'analyse des signaux à partir d'un pupitre.

L'ensemble de l'installation est représenté sur la planche 5. La maquette d'un ventilateur de soufflage à deux étages d'une centrale thermique d'E.D.F. est entraînée par un moteur asynchrone équipé d'un variateur de vitesse (300 tr/ $\mathrm{mn}, 1800 \mathrm{tr} / \mathrm{mn}$ ). A cause du bruit que fait la machine à grande vitesse, nous avons dû disposer un silencieux en amont et en aval de l'installation. Ainsi l'air pénètre d'abord dans un silencieux puis traverse la machine, passe dans une conduite de mesure où se trouve d'une part un diaphragme qui permet de déterminer les performances (pression, débit) et, d'autre part, un écran de tubes au moyen duquel on peut modifier le point de fonctionnement, l'air est ensuite restitué à l'atmosphère après avoir traversé le silencieux aval. La pale de mesure est représentée sur la planche 6 . La pale est entaillée à la base ce qui permet de faire varier ses fréquences propres. L'impulseur est fixé en bout de pale de façon à obtenir l'impulsion requise avec une faible charge de poudre. La mise à feu est réalisée au moyen d'une résistance chauffante.

La chaîne de mesures utilisée (cf. planche 7) est constituée par des ponts «B et $\mathrm{F}$ instruments » qui alimentent les jauges en courant constant. Les variations de tension qui apparaissent aux bornes des jauges sous l'effet du mouvement vibratoire, sont transmises par l'intermédiaire du collecteur, puis filtrées à la fréquence propre de flexion de la pale et enregistrées sur papier au moyen d'un enregistreur à galvanomètres. Les enregistrements ainsi obtenus permettent de déterminer le décrément logarithmique $\delta$ qui caractérise l'amortissement de la pale (cf. planche 7). La pale étant principalement excitée en flexion par limpulsion initiale et les jauges étant essentiellement sensibles aux contraintes de flexion, l'analyse harmonique montre que les signaux ne présentent pratiquement qu'une raie correspondant à la première fréquence propre de la pale, de sorte que leur filtrage permet de calculer $\delta$ avec plus de précision mais sans perte d'information.

L'amortissement est représenté en fonction de la période $\mathrm{Tr}$ définie de la façon suivante :

$$
\mathrm{T} r=\frac{\mathrm{TW}}{\mathrm{L}}=\frac{\mathrm{W}}{\mathrm{FL}}
$$

avec :

F : fréquence propre de flexion de la pale; 

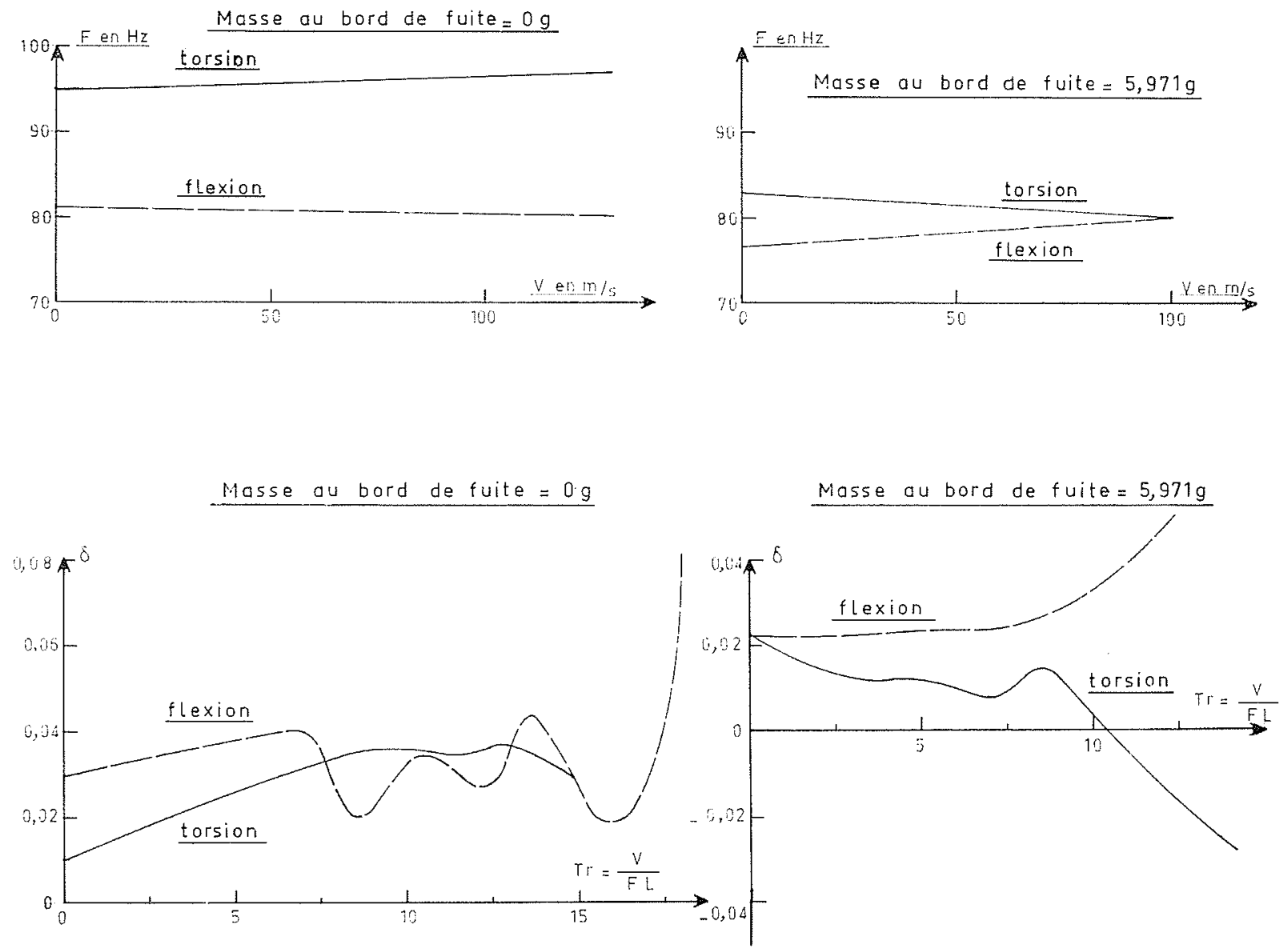

4/ Apparition du flottement en grille active.

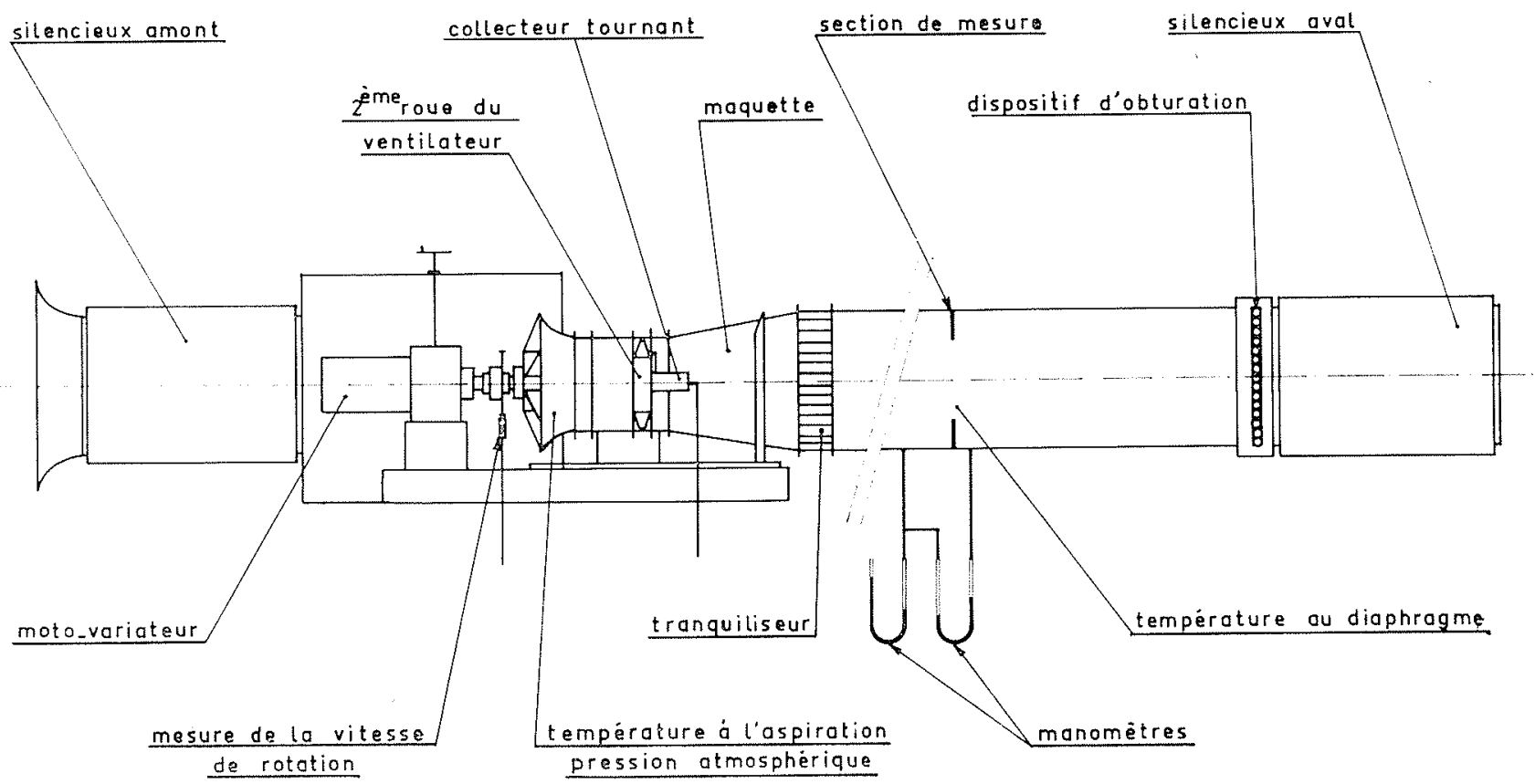

5/ Schéma de linstallation. 

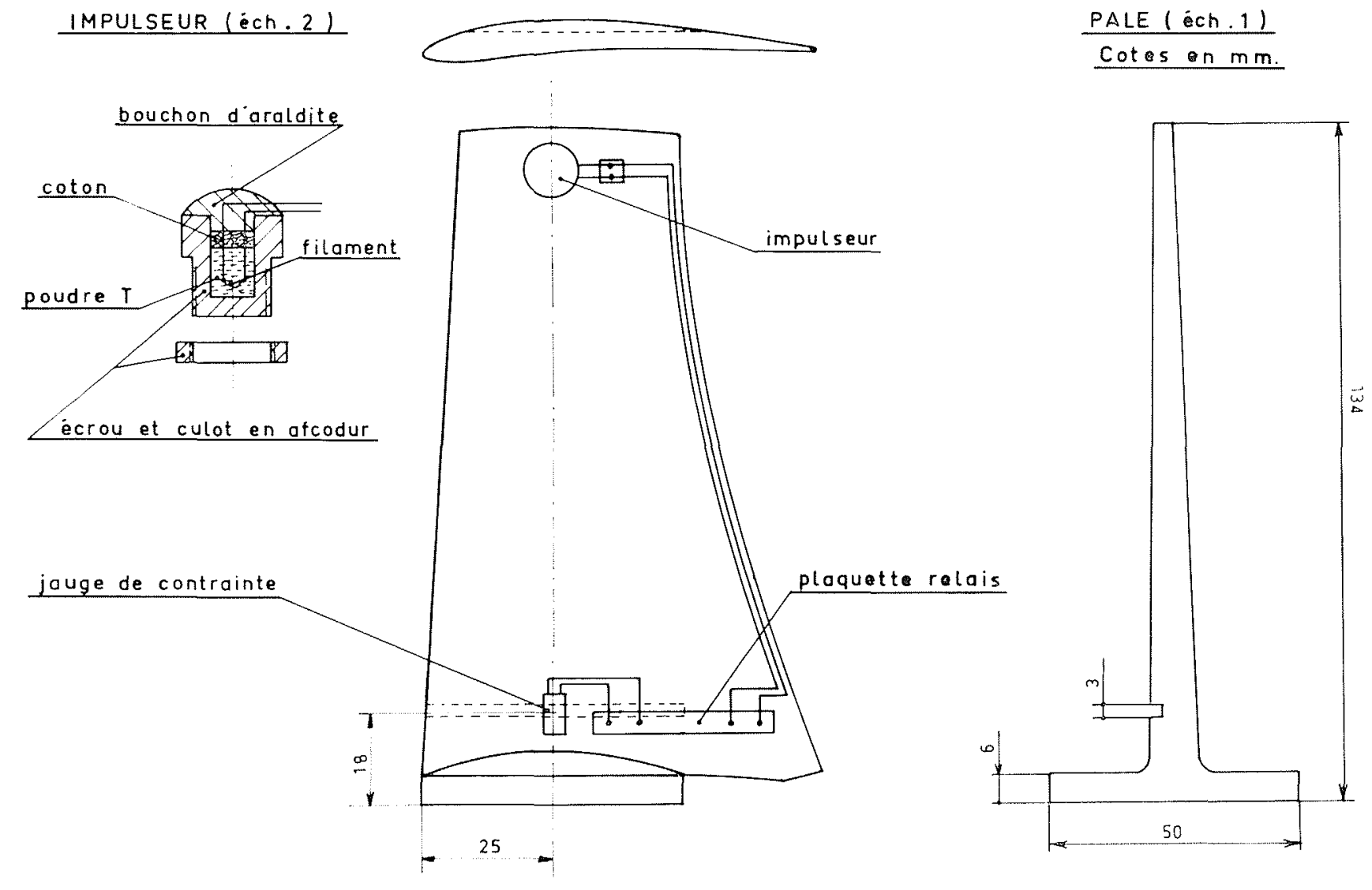

6/ Equipement de la pale entaillée.

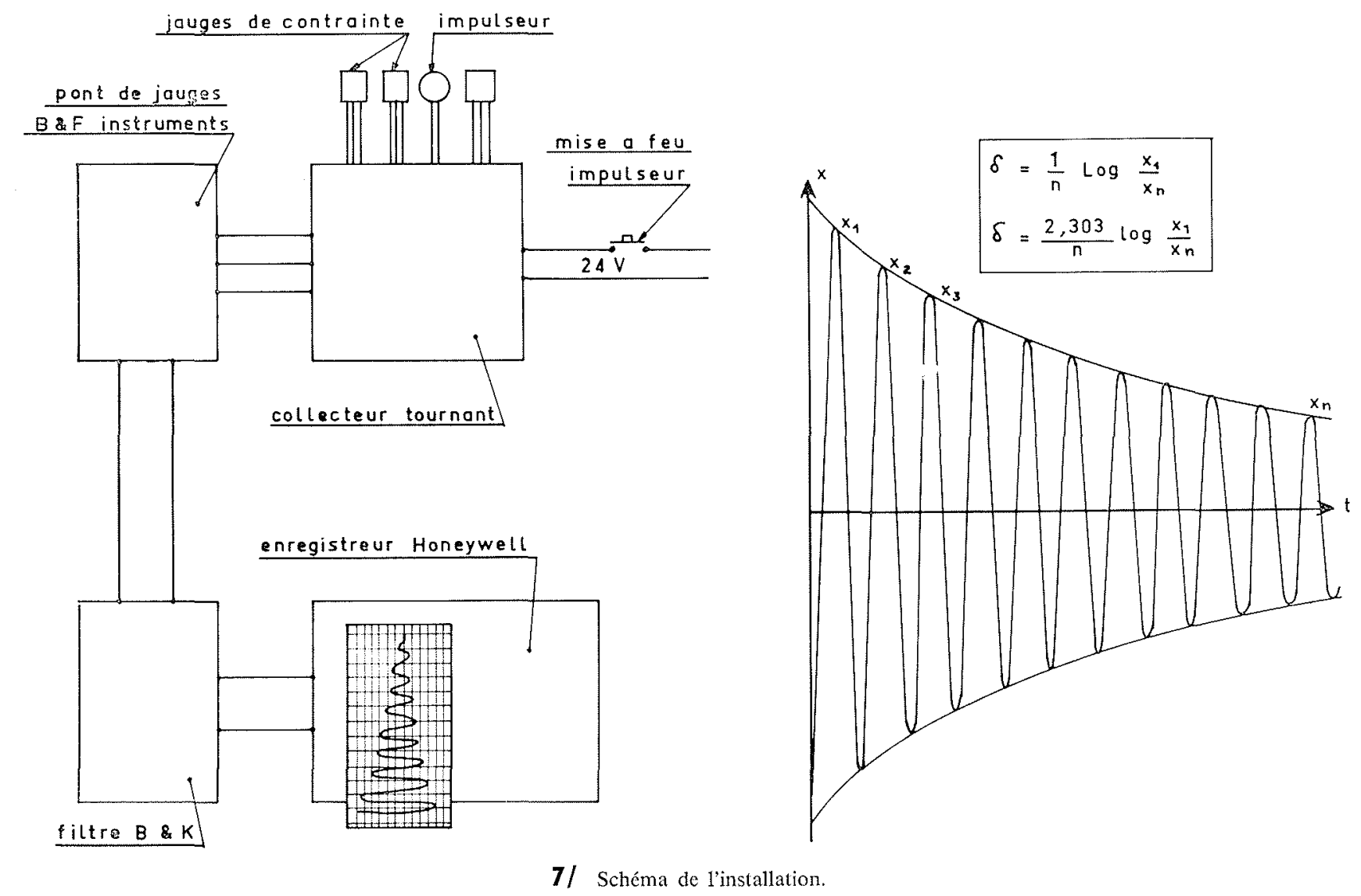


L: longueur de la corde de la pale $\left(45 \cdot 10^{-3} \mathrm{~m}\right)$;

$W$ : vitesse relative en bout de pale $\left(\mathrm{W}=\sqrt{V_{m}^{2}+u^{2}}\right.$;

$u$ étant la vitesse d'entraînement et $V_{m}$ la vitesse moyenne de débit).

La période réduite ainsi définie est l'équivalent du nombre de Strouhal. C'est une grandeur adimensionnelle qu'il est commode d'utiliser car elle représente à la fois les caractéristiques vibratoires et celles de l'écoulement en bout de pale.

Les résultats obtenus sont représentés sur les planches 8 à 12 . On voit que dans la gamme des périodes réduites que nous avons explorée l'amortissement aérodynamique est très important et conserve toujours un signe positif.

\section{Remorques sur les résultats obtenus}

Les résultats que nous avons obtenus sont relatifs à une aube entaillée de sorte que les autres pales de la roue ont des fréquences propres qui sont différentes de celles de la pale étudiée. Ceci permet d'éviter les battements dus aux excitations d'ordre mécanique qui sont transmises par le disque de la roue mais en même temps cela modifie les intéractions entre la pale étudiée et les aubes adjacentes. Toutefois, les amplitudes vibratoires de la pale étudiée

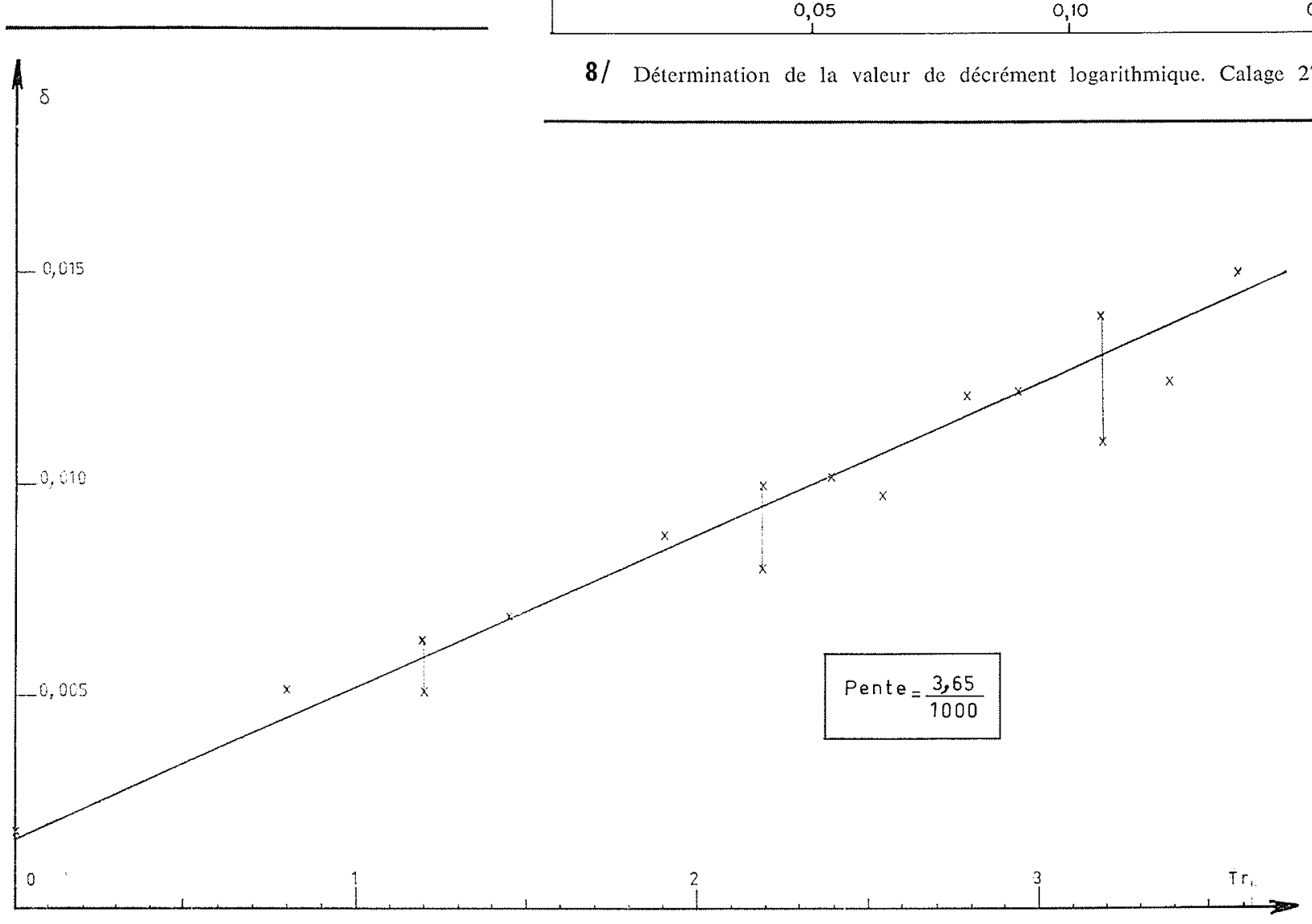

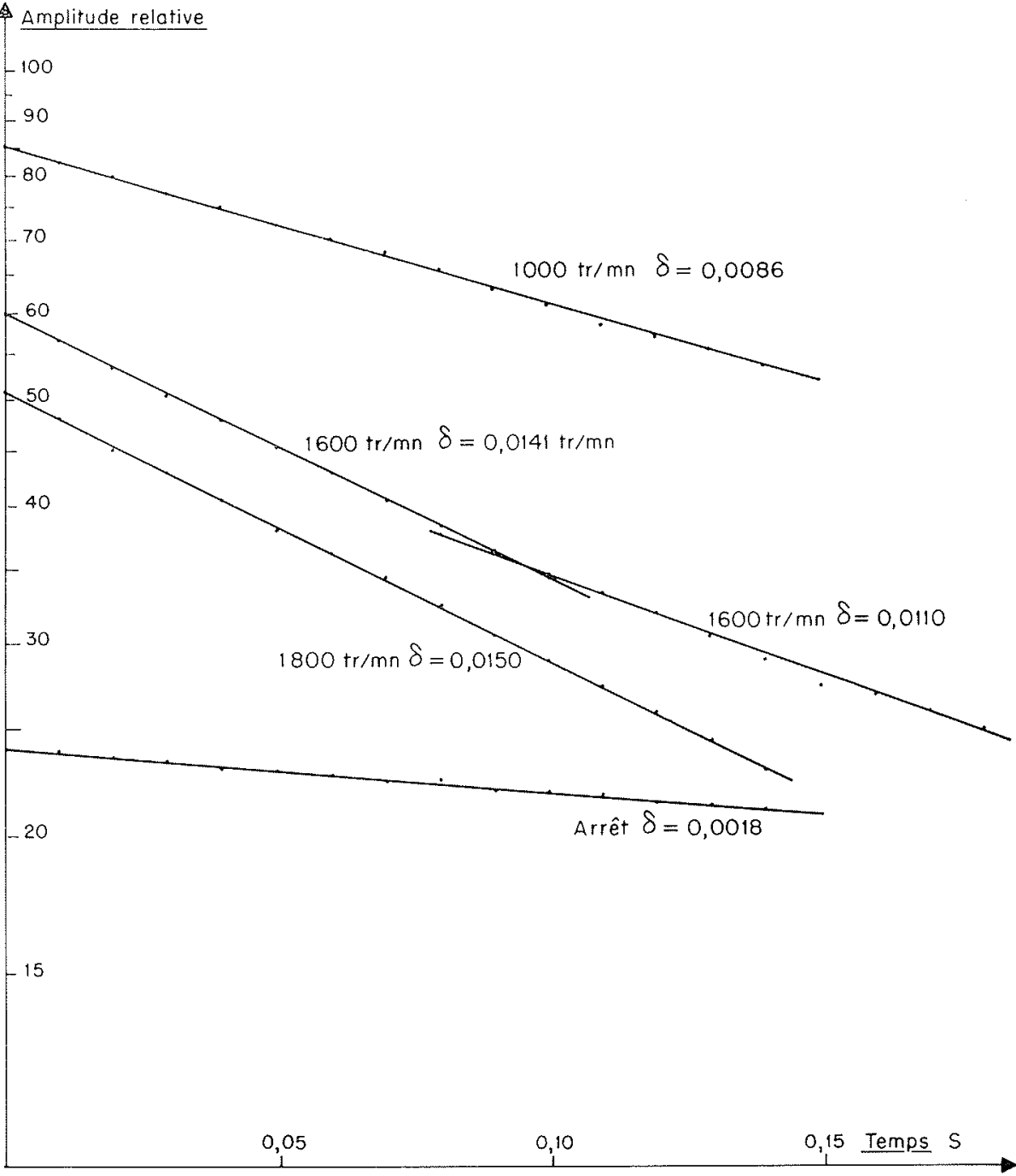

$8 /$ Détermination de la valeur de décrément logarithmique. Calage $27^{\circ} 30^{\prime}, \mu=0,3$

Décrément logarithmique en fonction de la fréquence réduite $\mu=0,3$ Calage $27^{\circ} 30^{\prime}$, 


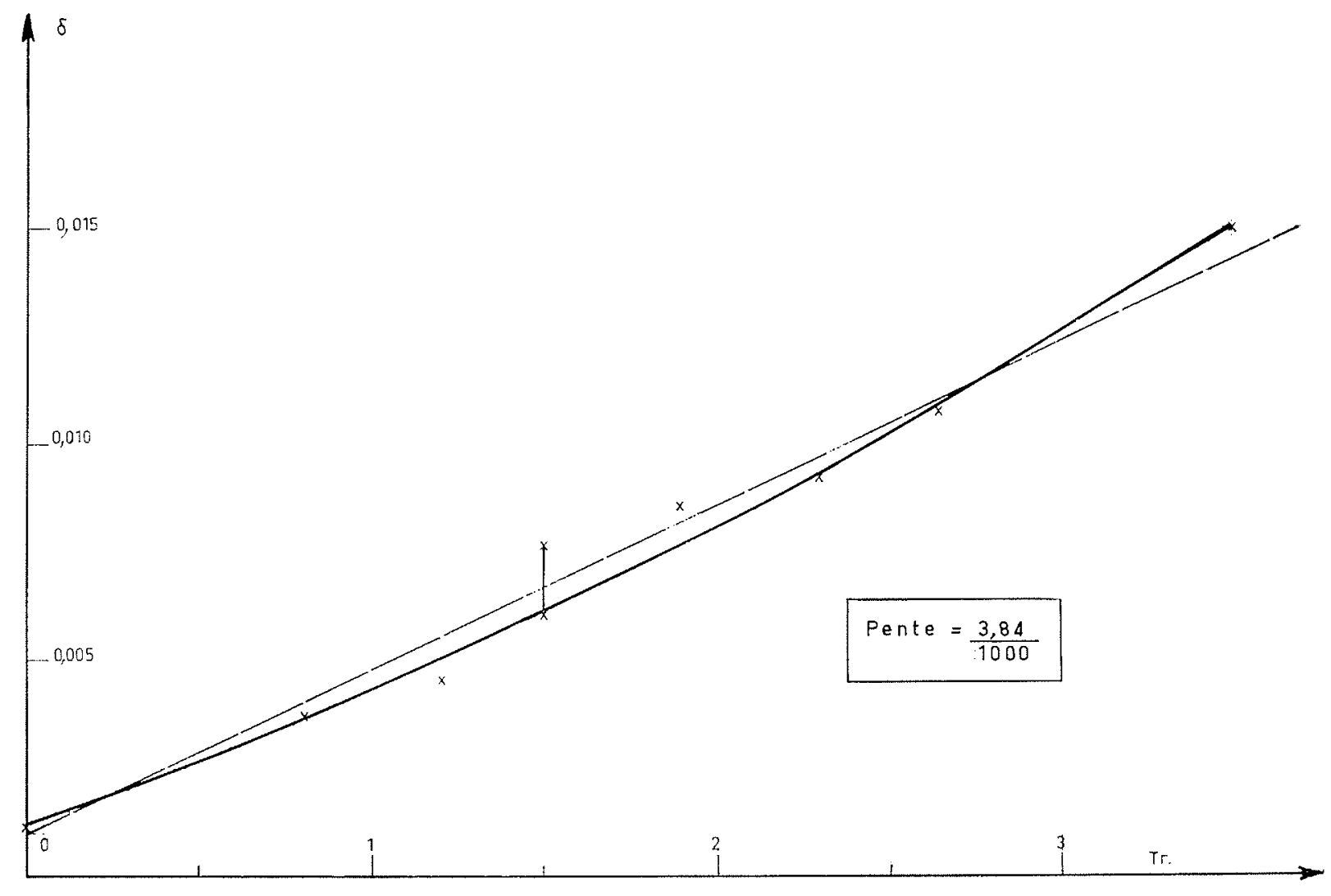

10/ Décrément logarithmique en fonction de la période réduite, $\mu=0,359$, calage $27^{\circ} 30^{\circ}$.
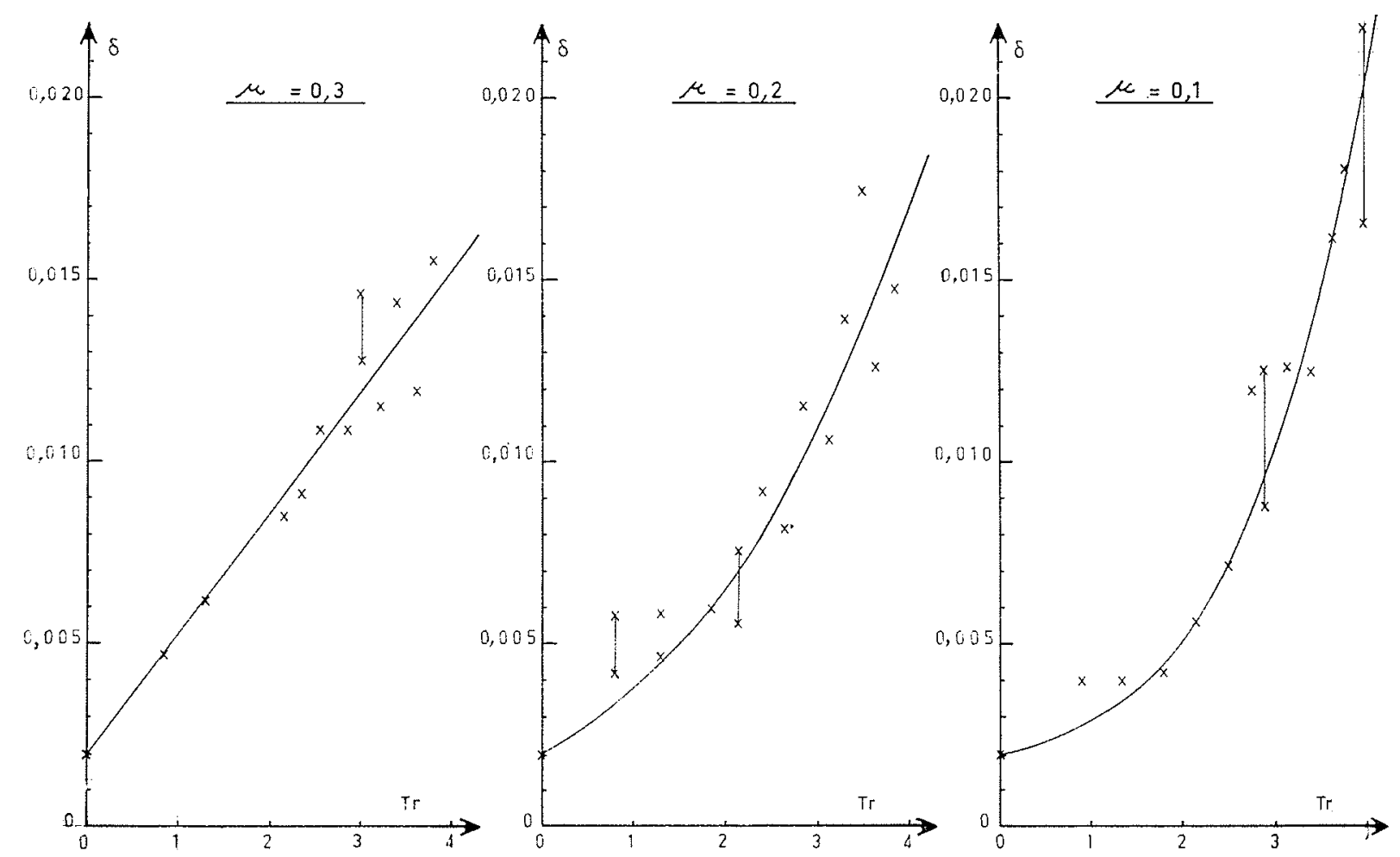

11/ Influence du point de fonctionnement de la machine. $\mathrm{F} \approx 380 \mathrm{~Hz}$, calage $45^{\circ}$. 

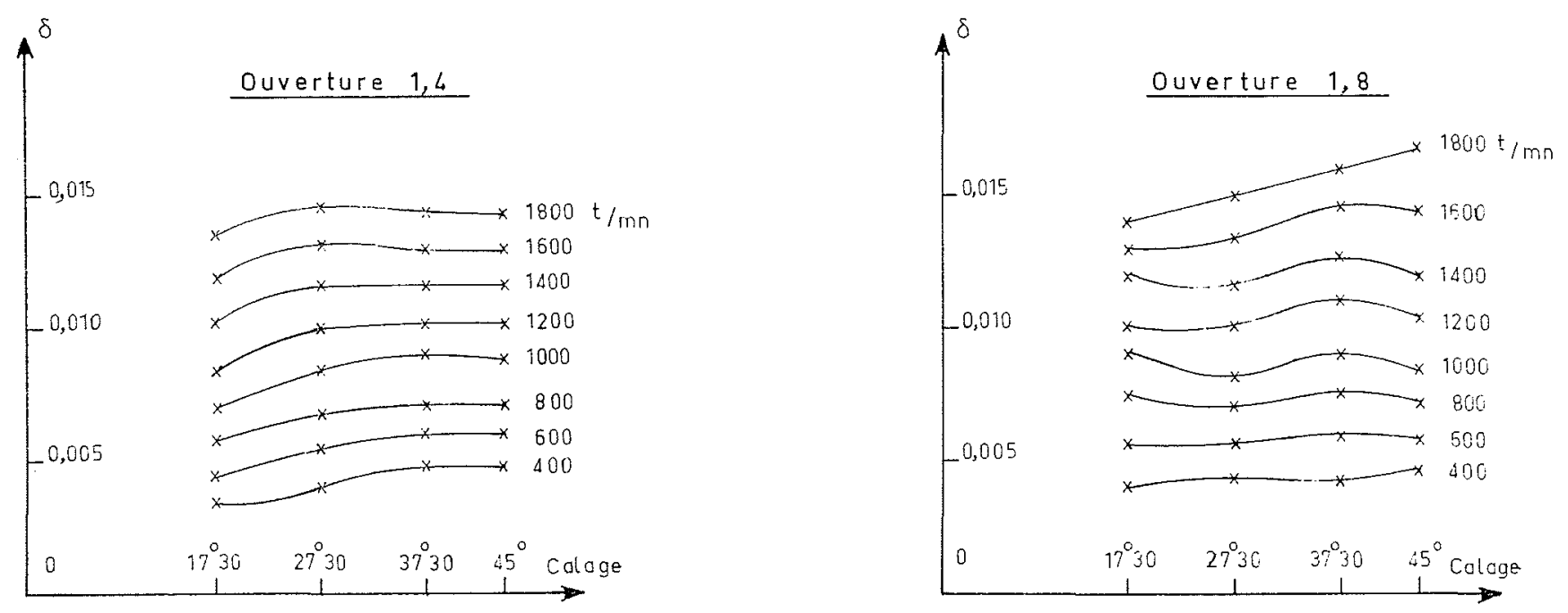

12/ Influnce du calage des pales pour des ouvertures et vitesses de rotation constantes.

étant faibles et comme on peut considérer que les aubes adjacentes sont pratiquement fixes, il en résulte que la différence de fréquence n'introduit sans doute pas d'effet important.

Le filtrage du signal issu des jauges permet d'obtenir plus facilement le coefficient d'amortissement relatif à la fréquence propre de fiexion de la pale entaillée. On a cependant constaté que les résultats obtenus étaient erronés pour la vitesse de rotation qui donnait une fréquence d'excitation par les sillages du distributeur entraînant la mise en résonance des aubes. La planche 13 montre les courbes de réponse des pales à ces excitations.

\section{Conclusions}

Les aubes de turbomachines sont soumises à des excitations d'origines diverses et dont l'importance est très mal connue. Dans le cas général il semble que l'amortissement aérodynamique joue un rôle essentiel et salutaire. Cependant, il serait souhaitable de pouvoir mieux connaître ces phénomènes, de façon quantitative, pour s'assurer que pour tous les points de fonctionnement de la machine et spécialement pour les régimes de fonctionnement hors adaptation, la combinaison des forces excitatrices et de l'amortissement conduisent à des contraintes acceptables. 


\section{Références}

[1] VON Karman and SEars. - Airfoil theory for non-uniform motion. Journal of the Aeronautical Sciences, vol. 5 (August 1938), $\mathrm{n}^{\circ} 10$.

[2] CARTA. - Stressing and vibrations for turbomachinery components. Short Course I.V.K. (November 1970), Belgium.

[3] LEgEndre (R.). - Amortissement aérodynamique des vibrations des aubes de turbomachines. Note technique ONERA $n^{\circ} 107$ (1967).
[4] Mecrer. - Etude préliminaire sur le flutter des aubes de compresseurs. Note technique ONERA $n^{\circ} 18$ (1953).

[5] Bellenot et Lalive D'Epinay. - Vibrations autoexcitées des aubes de turbomachines. Revue Brown Boveri, tome 37 (Octobre 1950).

[6] Rapports A.T.T.A.G., P. 95 et P. 102.

[7] Rapports A.T.T.A.G., P. 122 et P. 132

[8] Rapport A.T.T.A.G., P. 142.

\section{Discussion}

M. le Président remercie vivement M. Aureille d'avoir attiré l'attention sur l'importance de l'amortissement aérodynamique dans les turbo-machines et ouvre la discussion.

M. LEGENDRE, directeur technique de l'O.N.E.R.A. intervient, comme suit, à propos des travaux de l'A.T.T.A.G. auxquels les conférenciers ont fait allusion :

Je remercie Electricité de France d'avoir soutenu mon action à l'A.T.T.A.G. à l'époque où l'O.N.E.R.A. était invité à consacrer ses efforts à l'étude des missiles hypersoniques.

J'avais déjà pu interpréter avec l'aide du Professeur EIcheLBRENNER, le flottement observé dans un compresseur Brown-Boveri, dès 1950 , en montrant la possibilité du couplage de vibrations de torsion et de flexion; mais l'E.D.F. n'était pas obligée de me faire confiance lorsque je me proposais de vérifier expérimentalement mes prévisions à I'A.T.T.A.G. Je suis heureux qu'elle ait aujourd'hui prolongé mon action en abordant l'étude d'une véritable turbomachine.

Je suis convaincu qu'aucune turbo-machine, quel que soit le fluide, ne résisterait aux vibrations si aucun amortissement de celles-ci au sein du fluide ne majorait formidablement l'amortissement mécanique. Le malheur est que l'amortissement aérodynamique ne soit pas toujours positif et que, dès qu'il s'abaisse, souvent longtemps avant l'apparition d'un flottement franc, il permette l'établissement de vibrations destructrices sous l'effet des excitations forcées.

M. Aureille a montré que, pour les périodes réduites qui intéressent les machines de l'E.D.F., l'amortissement des vibrations de flexion pure, qu'il a séparées avec soin, est constamment positif et croissant, mais j'espère qu'il reconnaîtra que cette constatation n'est nullement rassurante, précisément parce que les risques de couplage de plusieurs modes ont été écartés. D'ailleurs, une cxpérience récente de I'A.T.T.A.G., menée en dehors du contrat de l'E.D.F., a interprété une avarie très ancienne d'un ventilateur d'alternateur. Un flottement caractéristique a été observé entre deux modes de fréquences très voisines à une période réduite très faible. Il est done incontestable qu'il a existé à l'E.D.F. des machines où l'amortissement aérodynamique était insuffisant pour assurer la sécurité, et j’ai bien l'impression que les derniers résultats présentés par $M$. AuREILLE prouvent que le ventilateur qu'il étudie entrera facilement en flottement s'il cesse de prendre des précautions pour n'exciter que la flexion, car une chute notable de l'amortissement se manifeste déjà. L'expérience de l'A.T.T.A.G., présentée par M. Aureille, montre qu'il suffit de rapprocher les fréquences de deux modes et de modifier très légèrement la répartition des masses pour amorcer un flottement destructeur. Il importe de ne pas masquer ce risque aux constructeurs.

La prévision théorique de l'amortissement aérodynamique est compliquée par l'effet des déphasages des vibrations des aubes voisines. Le déphasage le plus catastrophique semble correspondre à l'opposition de phase observée chez Brown-Boveri et pour Iaquelle les aubes «battent des mains 》 selon l'expression de MM. Brelenor et LaLive D'Espinay. L'étude des vibrations en opposition de phase est plus facile que celles des vibrations avec des phases sous multiples d'ordres plus élevés de $2 \pi$ car elle ne met en jeu que des fonctions elliptiques alors qu'il faut introduire les fonctions intégrales abéliennes d'un groupe kléinéen de Poincaré pour calculer les effets de déphasages plus faibles. M. LeClire peut commenter la méthode de résolution d'une équation intégrale qui n'est pas très simple, mais qui évite les complications de l'uniformisation de Poincaré que j'avais préférée pour l'étude des écoulements quasi stationnaires.

M. Aureille est bien d'accord avec les observations de M. LeGeNDRE; il a, d'ailleurs, noté dans sa communication que le couplage «torsion-flexion » de la grille plane essayée par l'A.T.T.A.G. pouvait conduire à la rupture de celle-ci. Toutefois, les derniers résultats de ses propres essais montrent que, malgré une diminution inquiétante de l'amortissement hydrodynamique, les fréquences de vibration sont essentiellement sur le premier mode de flexion.

M. Casaccr pose la question suivante

Les travaux consacrés aux vibrations d'une grille rectiligne de profils dans un écoulement à potentiel d'un liquide incompressible, précisent l'infuence des différents paramètres caractéristiques et notammment, celle du déphasage des vibrations des profils voisins. Ces calculs montrent que les forces et moments hydrodynamiques ou aérodynamiques peuvent dépendre fortement de la valeur de ce déphasage. Dans les essais que vient de nous présenter M. Aureille sur l'amortissement aérodynamique des vibrations d'aubes de turbomachines, les déphasages des vibrations synchrones des profils ont-ils pu être mesurés?

Nous nous préoccupons de cette question répond M. AUREILle. Mais pour effectuer les mesures de déphasage que vous évoquez, il faudrait notamment disposer de plusieurs aubes équipées de jauges; or, dans nos essais, nous avons dû entailler une aube de façon à avoir un phénomène plus pur. Nous nous proposons de revenir à une configuration où toutes les aubes seront autant que possible identiques; on pourra alors mesurer quel est l'effet du mouvement de l'aube principale sur les aubes adjacentes et réciproquement.

M. LeCLerC donne les précisions ci-après sur des essais qui vont être entrepris à la soufflerie de Modane sur une grille plane faiblement cambrée en vue d'étudier l'influence du déphasage des vibrations de chacune des aubes. L'analyse théorique montre que la mesure des efforts instationnaires sur les aubes voisines de la seule aube vibrante - ainsi que sur ces dernières — défnit parfaitement l'interaction pour un déphasage constant quelconque entre aubes consécutives.

Pour être tout à fait complet, ajoute M. Aurenlle, en ce qui concerne nos mesures sur une roue de ventilateur, je signale que certains résultats sont très difficiles à interpréter directement du fai de la tridimensionnalité de la roue. Les calculs évoqués par M. LECLFRC étant opérationnels, il nous a très aimablement proposé de lui confier les caractéristiques aérodynamiques d'une grille d'aube correspondant sensiblement à une coupe cylindrique représentative du ventilateur, de façon à pouvoir comparer ses calculs à nos résultats d'essais; pour que la comparaison soit encore plus instructive, on envisage de faire effectuer, à l'A.T.T.A.G., des essais sur une grills plane analogue à celle confiée à M. LECLERC; ainsi, on pourra procéder, d'une part, à la comparaison entre calculs ct essais en grille plane, et d'autre part, entre grille plane et machine réelle.

M. le Président clôt la discussion en remerciant tous ceux qui l'on animée. 